\title{
Is a mechanical-assist device better than manual chest compression? A randomized controlled trial
}

\author{
Chaiyaporn Yuksen' \\ Thidathit Prachanukool' \\ Kasamon Aramvanitch' \\ Nuttamon Thongwichit' \\ Kittisak Sawanyawisuth ${ }^{2-4}$ \\ Yuwares Sittichanbuncha' \\ 'Department of Emergency Medicine, \\ Faculty of Medicine Ramathibodi \\ Hospital, Mahidol University, \\ Bangkok, ${ }^{2}$ Department of Medicine, \\ Faculty of Medicine, Khon Kaen \\ University, ${ }^{3}$ Research Center in Back, \\ Neck Other Joint Pain and Human \\ Performance (BNOJPH), ${ }^{4}$ Sleep \\ Apnea Research Group, Khon Kaen \\ University, Khon Kaen, Thailand
}

Correspondence: Yuwares

Sittichanbuncha

Department of Emergency Medicine,

Faculty of Medicine, Ramathibodi

Hospital, Mahidol University, Bangkok,

10400, Thailand

Tel $+662201 \quad 1484$

Fax +6622012404

Email yuwares.sit@mahidol.ac.th
This article was published in the following Dove Press journal:

Open Access Emergency Medicine

29 August 2017

Number of times this article has been viewed

Background: Chest compression quality is a determinant of survival from sudden cardiac arrest. The CPR RsQ Assist Device (CPR RAD) is a new cardiopulmonary resuscitation device for chest compression. It is operated manually but it does not pull up on the chest on the up stroke. The aim of this study was to compare the CPR RAD with standard manual compression in terms of chest compression quality in a manikin model.

Methods: Participants were randomly assigned to either the device or manual chest compression group. Each participant performed a maximum of 4 minutes of hands-only compression with or without the device. During chest compression, the following quality parameters from the manikin were recorded: compression rate, compression depth, and correctness of hand position. Results: Duration of chest compression was significantly higher in device users compared with manual compression ( $223.93 \pm 36.53$ vs $179.67 \pm 50.81$ seconds; $P<0.001$ ). The mean compression depth did not differ in a statistically significant way between manual compression and device at 2 minutes $(56.42 \pm 6.42$ vs $54.25 \pm 5.32 ; P=0.052)$. During the first and second minutes, compression rate was higher in cases of standard compression $(133.21 \pm 15.95$ vs $108 \pm 9.45 ; P<0.001$ and $127.41 \pm 27.77$ vs $108.5 \pm 9.93 ; P<0.001)$. There was no statistically significant difference in the percentage of participants who employed compression that was too shallow or exhibited incorrect hand position.

Conclusion: The CPR RAD is more effective in chest compression compared with manual chest compression, as using the device led to better results in terms of fatigue reduction and correct compression rate than standard manual compression.

Keywords: chest compression, CPR RsQ Assist device, outcomes

\section{Introduction}

Chest compression is one of the most important procedures in cardiopulmonary resuscitation. Several factors should be considered during chest compression such as depth, rate, and location. The desired depth of chest compression is $5-6 \mathrm{~cm}$ at a rate of 100-120 times/minute. ${ }^{1,2}$ The quality of chest compression is directly associated with survival rate. . $^{3,4}$

The main problem that occurs in chest compression is fatigue on the part of the person conducting the compression. The American Heart Association recommends alternating compressors every 2 minutes. Several studies have shown that the automatic chest compression device (ACCD) is more effective than manual chest compression $^{5-7}$ and that the device has been shown to yield more effective chest compression rates than the standard manual method (69\% vs $35 \%) .{ }^{5}$ Several types of ACCDs (ie, mechanical resuscitation devices) are used in clinical practice. ${ }^{5-7}$ The 
CPR RsQ Assist Device (CPR RAD) is an ACCD that is operated manually but cannot make a fully reversible of the chest wall. Currently, there is no previous study in which randomized controlled trials comparing the CPR RAD and manual methods have been conducted.

\section{Methods}

This study was a randomized controlled trial conducted at the Department of Emergency Medicine, Mahidol University, Bangkok, Thailand. Medical personnel including medical students, physicians, nurses, emergency medicine technicians, and hospital staff were invited to participate in the study. Eligible participants were randomly assigned to either the manual or device group. The randomization method used sequentially numbered, opaque, sealed envelopes (SNOSE) and six-block randomization. The study protocol was approved by the Institutional Review Board, Mahidol University (MURA2015/602).

All participants gave informed consent prior to study participation and were given instruction on the CPR procedures outlined in the 2015 American Heart Association Guidelines for Cardiopulmonary Resuscitation and Emergency Cardiovascular Care. ${ }^{8}$ Then the participants were asked to perform chest compression on the Resusci Anne ${ }^{\circledR}$ SkillReporter ${ }^{\mathrm{TM}}$ for a maximum of 4 minutes or until fatigued. The manual group performed standard chest compression, while the device group performed chest compression using the CPR RAD with a set rate of 100 times/minute.

The data recorded in this study included duration of chest compression, chest compression rate, average chest compression depth, percentage of shallow compression, percentage of compression with incorrect hand position, and percentage of incomplete decompression. Shallow compression was defined as compression with a depth of $<50 \mathrm{~mm}$.

\section{Sample size calculation}

In a previous study, effective chest compression rates using the active compression device and standard manual method were shown to be $69 \%$ and $35 \%$, respectively. ${ }^{5}$ With a confidence of $95 \%$ and power of $80 \%$, the required study population was 78 subjects, with 39 in the manual group and 39 in the device group.

\section{Statistical analysis}

The characteristics of all participants in each group were compared by using descriptive statistics. Chest compression results were also compared between the device and manual groups using descriptive statistics where appropriate. All statistical analyses were performed by using STATA software version 14 (StataCorp LP, College Station, TX, USA).

\section{Results}

There were 80 participants in the study; 39 in the manual group and 41 in the device group. There were no statistically significant differences between the groups in terms of age, sex, CPR experience, and percentage of participants who had completed a CPR course (Table 1).

The average duration of chest compression was significantly longer in the device group than in the manual group (223.93 vs 179.67 seconds; $P<0.001$ ). The manual group had a significantly higher average chest compression rate at 1 and 2 minutes than the device group, while the average chest compression rate was significantly higher in the device group at minute 4 (90 vs 44.21 times/minute) as shown in Table 2.

Table I Clinical characteristics between the manual chest compression group and chest compression with automatic chest compression device group

\begin{tabular}{|c|c|c|c|}
\hline Factors & Manual group $(\mathrm{N}=39)$ & Device group $(\mathrm{N}=4 \mathrm{I})$ & $P$-value \\
\hline Age, years & $25.54(8.88)$ & $24.46(3.63)$ & 0.486 \\
\hline Female sex, n (\%) & $19(48.72)$ & $24(60.98)$ & $0.27 \mathrm{I}$ \\
\hline Body weight, kg & $62.22(14.47)$ & $6 I .54(14.8 I)$ & 0.835 \\
\hline Height, m & $1.66(0.08)$ & $1.65(0.08)$ & 0.346 \\
\hline Body mass index, $\mathrm{kg} / \mathrm{m}^{2}$ & $22.36(3.8)$ & $22.63(4.6)$ & 0.778 \\
\hline Occupation, $\mathrm{n}(\%)$ & & & 0.407 \\
\hline Medical student & $24(6 \mid .54)$ & $25(60.98)$ & \\
\hline Doctor & $8(20.5 \mathrm{I})$ & $13(31.71)$ & \\
\hline Nurse & $4(10.26)$ & I (2.44) & \\
\hline Paramedic & I (2.56) & $0(0)$ & \\
\hline Hospital staff & $2(5.13)$ & $2(4.88)$ & \\
\hline Completion of ACLS course, $n(\%)$ & $34(87.18)$ & $38(92.68)$ & 0.476 \\
\hline Experience with chest compression, $n(\%)$ & $30(76.92)$ & $35(85.37)$ & 0.334 \\
\hline
\end{tabular}

Note: Data presented as mean (SD) unless indicated otherwise.

Abbreviations: ACLS, advanced cardiac life support; SD, standard deviation. 
Table 2 Chest compression results between the manual chest compression group and chest compression with automatic chest compression device group

\begin{tabular}{|c|c|c|c|}
\hline Chest compression outcomes & $\begin{array}{l}\text { Manual group } \\
(n=39)\end{array}$ & $\begin{array}{l}\text { Device group } \\
(n=41)\end{array}$ & $P$-value \\
\hline Time to stop chest compression, seconds & I $79.67(50.8 \mathrm{I})$ & $223.93(36.53)$ & $<0.001$ \\
\hline \multicolumn{4}{|l|}{ Compression rate/minute } \\
\hline $0-I$ minute & |27.4| (27.77) & $108(9.45)$ & $<0.001$ \\
\hline $\mathrm{I}-2$ minute & $90.74(51.57)$ & $108.55(9.93)$ & $<0.001$ \\
\hline $2-3$ minute & $44.21(52.89)$ & $97.9(30.74)$ & 0.190 \\
\hline $3-4$ minute & & $90(39.11)$ & $<0.001$ \\
\hline Compression depth after 2 minute, $\mathrm{mm}$ & $56.42(6.42)$ & $54.25(5.32)$ & 0.052 \\
\hline Overall compression depth, $\mathrm{mm}$ & $49.21(6.04)$ & $46.35(6.40)$ & 0.045 \\
\hline \multicolumn{4}{|l|}{ Percentage of shallow compression } \\
\hline $0-I$ minute & $25.90(35.55)$ & $34.39(35.21)$ & 0.273 \\
\hline $\mathrm{I}-2$ minute & $46.49(39.88)$ & $61.22(41.77)$ & 0.159 \\
\hline $2-3$ minute & $55.14(44.46)$ & $72.69(37.81)$ & 0.343 \\
\hline $3-4$ minute & $62.18(44.21)$ & $80.23(33.70)$ & 0.106 \\
\hline \multicolumn{4}{|c|}{ Percentage of compression with incorrect hand position } \\
\hline $0-I$ minute & $10.43(25.37)$ & 31.41 (4I.59) & 0.632 \\
\hline $\mathrm{I}-2$ minute & $18.53(31.67)$ & $30.82(41.64)$ & 1.000 \\
\hline $2-3$ minute & 17.18 (33.78) & $23.37(37.53)$ & 1.000 \\
\hline $3-4$ minute & $23.44(39.87)$ & $19.29(35.24)$ & 0.642 \\
\hline \multicolumn{4}{|l|}{ Percentage of incomplete release } \\
\hline $0-I$ minute & $6.78(18.59)$ & $0.90(3.75)$ & 0.999 \\
\hline $\mathrm{I}-2$ minute & $7.13(19.82)$ & $2.32(9.92)$ & 0.999 \\
\hline $2-3$ minute & $6.25(20.35)$ & $1.92(10.59)$ & 0.999 \\
\hline $3-4$ minute & $5.5 I(15.43)$ & $0.16(0.8 I)$ & 0.999 \\
\hline
\end{tabular}

Note: Data presented as mean (SD).

Abbreviation: SD, standard deviation.

There was no significant difference in average depth of chest compression between the two groups at 2 minutes ( 56.42 vs $54.25 \mathrm{~mm} ; P=0.052$ ). At 4 minutes, the average depth of chest compression was deeper in the manual group than in the device group ( $49.21 \mathrm{vs} 46.35 \mathrm{~mm} ; P=0.045$ ) as shown in Table 2 . There was no difference between the two groups in the following areas at 4 minutes: percentage of shallow compression, incorrect location of chest compression, and incomplete release of chest compression (Table 2).

\section{Discussion}

Use of the CPR RAD led to better chest compression procedures than the manual method in terms of continuity and appropriate compression rate. The manual method had better compression depth than the CPR RAD, but other indicators of compression quality were comparable including shallow compression, incorrect hand position, and incomplete release.

Participants in the manual group stopped chest compression at a significantly shorter duration than those in the device group (Table 2). The device group almost reached the 4-minute study limit (223 seconds), while the manual group stopped chest compression at 3 minutes (179 seconds). However, the average compression rate in the manual group decreased dramatically after 3 minutes (from 90 to
44 times/minute). The American Heart Association recommends rotating compressors every 2 minutes when using the manual method. According to one study, $>90 \%$ of CPR $\mathrm{PRO}^{\circledR}$ device users preferred the device as it results in less fatigue. ${ }^{6}$

The appropriate rate for chest compression is between 100 and 120 times/minute. The CPR RAD is helpful in ensuring a correct and consistent compression rate. The manual group had a higher-than-recommended average compression rate at the first minute (133 times/minute), but the rate decreased over the duration of the procedure. On the other hand, the device group had quite consistent rates for the first 3 minutes. At the 4th minute, the compression rate was somewhat lower than the recommended compression rate (90 times/minute). Therefore, the device contains a metronome but the manual group did not have the metronome. Use of the CPR RAD also yielded a more adequate compression rate compared to that of an automatic ACCD reported in a previous study (108 vs 139 times/minute). ${ }^{7}$ Unlike other ACCDs, the CPR RAD has a red signal that blinks at a regular interval, which may help to ensure regular and adequate compression rates.

Another factor that may contribute to effective chest compression is the depth of the compression. Adequate circulatory perfusion requires a compression depth of 5-6 cm. ${ }^{1,2}$ Both 
the groups exhibited appropriate compression depth after 2 minutes, but after that, the average depths in both the groups were lower than the desired level (Table 2). These findings may indicate participants' fatigue, even when using the chest compression device. In fact, compression depth was significantly lower in the device group than in the manual group on average. The manual group seemed to exhibit stronger compressions, particularly in the first 2 minutes (Table 2).

Regarding other results, there were comparable percentages of shallow compression, incorrect hand position, and incomplete release in both the groups (Table 2). The manual group exhibited better performance in terms of shallow compression and correct position than the device group. These findings indicate that manual compression yielded better results in terms of landmark accuracy and harder compression. Unlike the CPR RAD, users of the CPR PRO have been shown to have better hand position than those employing manual chest compression (4.0\% incorrect hand position in the CPR PRO). ${ }^{6}$ The device is round in shape, which may be confusing to users attempting to accurately place it over the chest wall (Figure 1). The use of ACCD may require more practice than manual compression. However, the device had a non-significantly better outcome in incomplete release than the manual group. The device enabled greater regularity in terms of rhythm of chest compression due to the presence of the red signal, resulting in more appropriate chest-release time than the manual method (Table 2).

We would still recommend rotating compressors performing chest compression with the CPR RAD after 3 minutes. This is because the compression rate dropped below 100 times/minute and the percentage of shallow compression was high at $80 \%$ after 3 minutes. Because the CPR RAD is a semi-ACCD, it still requires physical exertion on the part of the rescuers. Even with the red signals from the machine, the compression rates were not adequate or were not in time

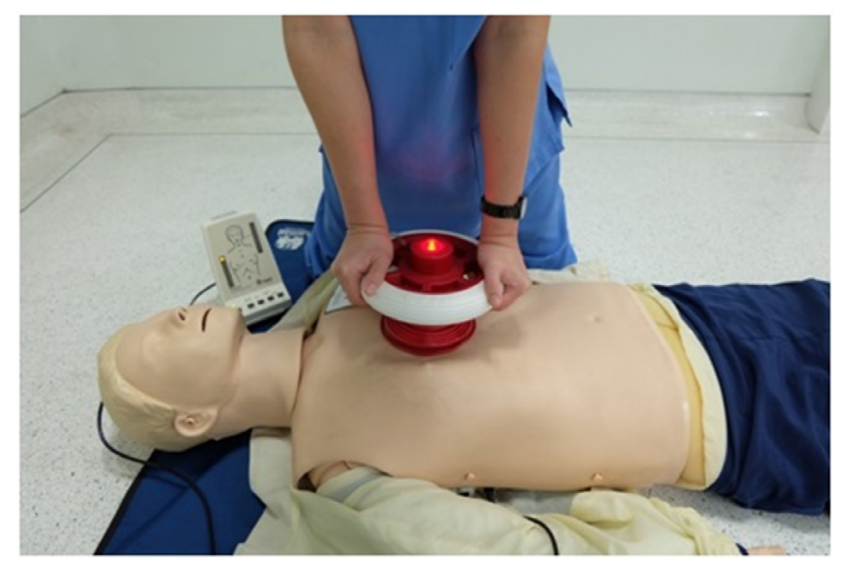

Figure I CPR RsQ Assist Device (CPR RAD) used for chest compression. with all of the signals. These findings indicated fatigue on the part of the participants, even with the ACCD.

There are some limitations in this study. First, the results of this study may not apply in real clinical situations. The results were recorded using a manikin, but may be used as preliminary data for further research regarding clinical use of the CPR RAD. Second, participants varied in terms of experience, with most being medical students. As a result, some results were not consistent with the standard recommendations. However, some results, such as shallow compression and correct position, were better in the manual group as mentioned earlier. The CPR RAD device may require more practice or training than the manual procedures. Finally, these results only apply to the CPR RAD and not other kinds of devices. Some CPR techniques may be useful in the combination with the CPR RAD such as using inclined step stool. ${ }^{9,10}$

\section{Conclusion}

The CPR RAD is more effective in chest compression compared with manual chest compression, as using the device led to better results in terms of fatigue reduction and correct compression rate than standard manual compression.

\section{Acknowledgments}

The authors would like to thank Prof. James A Will for his kind manuscript English editing via Publication Clinic KKU, Thailand, the Thailand Research Fund (IRG 5780016); Faculty of Medicine, Khon Kaen University, Thailand (Grant Number RG59301); and the TRF Senior Research Scholar Grant from the Thailand Research Fund (TRF grant number RTA5880001) and the Higher Education Research Promotion and National Research University Project of Thailand, Office of the Higher Education Commission, Thailand, through the Health Cluster (SHeP-GMS), Khon Kaen University.

\section{Disclosure}

The authors report no conflicts of interest in this work.

\section{References}

1. Kramer-Johansen J, Myklebust H, Wik L, et al. Quality of out-of-hospital cardiopulmonary resuscitation with real time automated feedback: a prospective interventional study. Resuscitation. 2006;71(3):283-292.

2. Edelson DP, Litzinger B, Arora V, et al. Improving in-hospital cardiac arrest process and outcomes with performance debriefing. Arch Intern Med. 2008;168(10):1063-1069.

3. Hoek TL, Becker LB, Abella BS. Rescuer fatigue during actual inhospital cardiopulmonary resuscitation with audiovisual feedback: a prospective multicenter study. Resuscitation. 2009;80(9):981-984.

4. Manders S, Geijsel FE. Alternating providers during continuous chest compressions for cardiac arrest: every minute or every two minutes? Resuscitation. 2009;80(9):1015-1018. 
5. Kurowski A, Czyzewski L, Bogdanski L, Zasko P, Karczewska K, Szarpak L. Quality of chest compression with CardioPump CPR compared to single rescuer standard BLS. Am J Emerg Med. 2015;33(1):114-115.

6. Kovic I, Lulic D, Lulic I. CPR PRO ${ }^{\circledR}$ device reduces rescuer fatigue during continuous chest compression cardiopulmonary resuscitation: a randomized crossover trial using a manikin model. J Emerg Med. 2013;45(4): 570-577.

7. Fischer $\mathrm{H}$, Neuhold $\mathrm{S}$, Zapletal B, et al. A manually powered mechanical resuscitation device used by a single rescuer: a randomised controlled manikin study. Resuscitation. 2011;82(7):913-919.
8. American Heart Association Guidelines Update for Cardiopulmonary Resuscitation and Emergency Cardiovascular Care. Circulation. 2015;13(S2):S414-S435.

9. Yun SW, Lee BK, Jeung KW, et al. The effect of inclined step stool on the quality of chest compression during in-hospital cardiopulmonary resuscitation. Am J Emerg Med. 2014;32(8):851-855.

10. Chung TN, Bae J, Kim EC, et al. Induction of a shorter compression phase is correlated with a deeper chest compression during metronomeguided cardiopulmonary resuscitation: a manikin study. Emerg Med J. 2013;30(7):551-554.
Open Access Emergency Medicine

\section{Publish your work in this journal}

The Open Access Emergency Medicine is an international, peerreviewed, open access journal publishing original research, reports, editorials, reviews and commentaries on all aspects of emergency medicine. The manuscript management system is completely online and includes a very quick and fair peer-review system, which is all

\section{Dovepress}

easy to use. Visit http://www.dovepress.com/testimonials.php to read real quotes from published authors. 